$\mathrm{IC} / 96 / 51$

hep-th/9604063

March 1996

\title{
Screening in Two-dimensional QCD
}

\author{
E. Abdalla ${ }^{a}$, R. Mohayaee ${ }^{b}$ and A. Zadra ${ }^{c}$ \\ International Centre for Theoretical Physics, Trieste, Italy \\ ${ }^{a}$ elcio@ictp.trieste.it \\ ${ }^{b}$ mohayaee@ictp.trieste.it \\ c azadra@uspif.if.usp.br
}

\begin{abstract}
We discuss the issue of screening and confinement of external colour charges in bosonised two-dimensional quantum chromodynamics. Our computation relies on the static solutions of the semi-classical equations of motion. The significance of the different representations of the matter field is explicitly studied. We arrive at the conclusion that the screening phase prevails, even in the presence of a small mass term for the fermions. To confirm this result further, we outline the construction of operators corresponding to screened quarks.
\end{abstract}

\footnotetext{
${ }^{1}$ Permanent address: Instituto de Física-USP, C.P. 20516, S. Paulo, Brazil.
} 


\section{Introduction}

The issue of confinement of fundamental constituents of matter is a long-standing problem of theoretical physics whose solution has evaded full comprehension up to now. However, significant progress has been made towards making a clear distinction between the apparently related phenomena of screening and confinement. In fact, in two-dimensional quantum electrodynamics, the original naive definition of confinement, as the absence of a pole in the gauge-invariant fermionic two-point function, has been replaced by a more subtle formulation which depends on the introduction of flavour quantum numbers 3 , 国. In the screening phase, any attempt to separate a pair of charged particles, bound by a weak potential, leads to vacuum polarisation which prevents the recognition of the individual charges. The introduction of the above mentioned flavour quantum numbers overcomes this problem: such new quantum numbers are distinguishable in the screening case, but not in the confining picture.

In this case [1], a rich physical structure exists, and the issue of confinement is satisfactorily settled [2]. The first step towards understanding the problem in the abelian case is taken by studying the potential created by two opposite charged particles $(q, \bar{q})$ a distance $L$ apart. We determine which of the two phases, i.e. screening or confinement, dominates by searching for the solitonic solutions of the classical field equations. These equations are obtained for QED coupled to the external charges (i.e. charges described by dynamical but classical quarks). In the solitonic solutions the sum of the non-vanishing external and fundamental charges has to be zero [6]. A simple mechanical problem equivalent to this setting has been introduced in [2] (see also (画). In this picture, the solitonic states are equivalent to the configuration in which one of the charged particles stays at the origin while the other approaches infinity. If the corresponding energy is finite, we have a single charge which is screened. If the energy grows linearly with distance the particle is confined. The latter phenomenon arises in the massive Schwinger model.

In two-dimensional quantum chromodynamics the situation is different and more involved [7]. One of the main differences concerns the vacuum structure. In the Schwinger model, the vacuum structure arises from a unitary operator which acts as a phase on the physical Hilbert space, characterising the ground state of the theory, which is 
infinitely degenerated and is labelled by a continuous parameter $\theta$, $0 \leq \theta<2 \pi$. In the massive case, the vacuum parameter modifies the dynamics by entering the theory explicitly at the lagrangian level. In that case, the issue of confinement can only be settled after a knowledge of the value of the $\theta$ parameter. In general there is a confining term, in the expression for the potential, whose scale is dictated by the fermion mass parameter. This term disappears for $\theta=\pi$ and for heavy quarks, leaving only a strong version of the screening term. In $\mathrm{QCD}_{2}$, the $\theta$ vacuum-parameter is effectively replaced by a dynamical field in the resulting semi-classical equations of motion, and the task of distinguishing between the screening and the confinement phase is a more subtle one.

In both theories, however, the question of screening versus confinement can be reformulated in terms of the equations of motion of the electric field and the bosonised matter field. The answer then depends on whether the Coulomb interaction survives, or whether the Higgs phase dominates 88 .

Our aim in this article is to answer the following questions: given two-dimensional QCD with an arbitrary number of dynamical fermions coupled to background charges (possibly fractional), are these background charges screened or confined? Moreover, if these charges are screened, can one define exotic states [4] carrying flavour quantum numbers? Our results show that the background charge density is always screened. This result is confirmed by our further analysis of the exotic states.

We start by reviewing the Schwinger model, summarising some of the earlier results in order to set up the language and outlining the construction of the exotic states. The procedure will be the paradigm of our discussion of two-dimensional QCD. In Section 3, we study the non-abelian problem. We recall the massless case where the system is in a screening phase. Then, we turn to the more complicated case of massive two-dimensional QCD. We consider all the possible solutions of the classical equations of motion and show that the ones which satisfy the requirement of finiteness of energy and suitable boundary conditions lead to screening. In Section 4, we study QCD with fermions in higher representations. We point out how the theory is affected by the change of representation and re-establish the results obtained in Section 3. In Section 5, we discuss the construction of exotic states which correspond to operators that carry flavour quantum 
numbers but no colour charge [3]. Thus, we provide a further evidence for the screening phase. We discuss our conclusions and further open problems in the last section.

\section{The Schwinger model}

In this section, we review the Schwinger model coupled to a pair of background charges $(q, \bar{q})$ at positions $\left(-\frac{L}{2}, \frac{L}{2}\right)$. This introduces an additional constant electric field in the space between the two charges. We follow reference [2] and start by writting the contribution of the two probe particles to the charge density, i.e.

$$
J^{0}=q\left[\delta\left(x-\frac{L}{2}\right)-\delta\left(x+\frac{L}{2}\right)\right]=-\frac{e}{\sqrt{\pi}} \frac{\partial Q}{\partial x},
$$

where

$$
Q=\frac{-q}{e} \sqrt{\pi}\left[\Theta\left(x-\frac{L}{2}\right)-\Theta\left(x+\frac{L}{2}\right)\right],
$$

and $\Theta$ is the step function. In the bosonised form of the action, or equivalently in the effective action, this background density contributes through the anomaly term, corresponding to the meson mass term. That is to say, the lagrangian is

$$
\mathcal{L}=\frac{1}{2}\left(\partial_{\mu} E\right)^{2}-\frac{e^{2}}{2 \pi}(E-Q)^{2}-2 m^{2}[1-\cos (2 \sqrt{\pi} E+\theta)]
$$

where $E$ is the meson field, $e$ is the electric charge unit and $m$ is the electron mass. The modification of the lagrangian brought about by the $\theta$ vacuum is transparent in the mass term. The inter-charge potential energy is obtained after finding the field configurations which minimise the hamiltonian. By considering time-independent fields we obtain the hamiltonian

$$
H=\int d x\left\{\frac{1}{2} E^{\prime 2}+\frac{e^{2}}{2 \pi}(E-Q)^{2}+2 m^{2}[1-\cos (2 \sqrt{\pi} E+\theta)]\right\} .
$$

Thus, we may use an analogy with a simple mechanical problem. In this setting, $x$ is the time evolution variable, $E$ is the position of a particle and $H$ is the action. Minimising this action leads to the Euler-Lagrange equations. 
The inter-charge potential is defined by the change in energy caused by the presence of the probe charges (i.e. $V(L)=H(L)-H(0)$ ). One can see that this potential is given by

$$
\frac{d V(L)}{d L}=-\frac{e q}{2 \sqrt{\pi}}[E(L / 2)+E(-L / 2)]+\frac{q^{2}}{2},
$$

where we impose the boundary conditions $E( \pm \infty)=0$ and require the continuity of $E$ and its derivative $E^{\prime}$ at $x= \pm L / 2$. For massless fermions, it is easy to solve the problem by quadratures and one finds a screening potential,

$$
V(L)=\frac{q^{2} \sqrt{\pi}}{2 e}\left(1-e^{-e L / \sqrt{\pi}}\right) .
$$

For non-vanishing fermion mass the computation is similar, although the integral cannot be computed in closed form. In the approximation for heavy fermions, the particle moves in the proximity of the origin, around which the solution is expanded. We obtain for $\theta=0$ and for small separations

$$
V(L)=\frac{e^{2} q^{2}}{2 \pi \alpha^{3}}\left(1-e^{-\alpha L}\right)+\frac{q^{2}}{2}\left(1-\frac{e^{2}}{\pi \alpha^{2}}\right) L,
$$

where $\alpha=\sqrt{e^{2} / \pi+8 \pi m^{2}}$. Hence, a confining term appears in the potential.

Next, we reconsider the hamiltonian (4) in the general case when the mass $m$ and the $\theta$-parameter are non-vanishing. We must solve the equation of motion of $E(x)$, such that $E$ approaches its vacuum expectation value at $\pm \infty$. That is to say $E( \pm \infty)=E_{\theta}$ where the constant $E_{\theta}$ is the solution of the equation which minimises the potential, i.e.,

$$
\sin \left(2 \sqrt{\pi} E_{\theta}+\theta\right)=-\frac{1}{4 \pi \sqrt{\pi}} \frac{e^{2}}{m^{2}} E_{\theta} .
$$

For large mass, $m>>e$, we can solve the equations perturbatively. Defining $F$ as the deviation of $E$ from the vacuum state,

$$
E=F+E_{\theta},
$$

we find the equation of motion of $F$ to be,

$$
F^{\prime \prime}-\left(\frac{e^{2}}{\pi}+8 \pi m^{2} \cos \left(2 \sqrt{\pi} E_{\theta}+\theta\right)\right) F=-\frac{e^{2}}{\pi} Q .
$$


Finally, using the boundary conditions $F( \pm \infty)=0$, we compute the inter-charge potential energy. In the case $q=e$, we find

$$
V(L) \simeq \frac{e^{4}}{2 \pi \alpha^{3}}\left(1-e^{-\alpha L}\right)+\frac{e^{2}}{2 \pi}\left(1-\frac{e^{2}}{\pi \alpha^{2}}\right)(\theta-\pi) L .
$$

For $\theta=\pi$, the confining term vanishes and we obtain a purely screening potential. In such a case screening plays a rather dominant rôle, being more effective than in the massless case.

However, this semi-classical description of the inter-charge energy eventually fails, due to pair production. Pair production will always lead to screening when the external charges are integer multiples of the fundamental charges. Similarly, in the Wilson loop approach, where the perimeter versus area law presumably distinguishes between screening and confinement, the presence of dynamical fermions implies the perimeter law. Therefore, one needs a more elaborate formulation. The question as to whether there is confinement can be definitely answered if we introduce further quantum numbers into the theory and verify whether these new quantum numbers can be measured asymptotically (screening) or not (confinement).

Such a task has been accomplished in two-dimensional QED where flavour quantum numbers were introduced. If the fermions belong to a flavour $\mathrm{SU}(\mathrm{k})$ representation (abelian) then bosonisation leads to the hamiltonian

$$
\begin{aligned}
H & =\int\left(\mathcal{H}_{0}+V\right), \\
\mathcal{H}_{0} & =\frac{1}{2} \dot{E}^{2}+\frac{1}{2} E^{\prime 2}+\frac{1}{2} \sum_{i=1}^{k-1}\left(\dot{\phi}_{i}^{2}+{\phi_{i}^{\prime}}^{2}\right), \\
V & =\frac{1}{2} \frac{k e^{2}}{\pi} E^{2}-2 m^{2} \sum_{f} \cos \left(2 \sqrt{\frac{\pi}{k}} E+2 \sqrt{\pi} \chi_{f}+\theta\right),
\end{aligned}
$$

where $\chi_{f}=\sum_{i} \phi^{i} \tau_{f f}^{i}$. For $\mathrm{SU}(\mathrm{k}), \sum_{f=1}^{k} \chi_{f}=0$. The fields $\phi^{i}$ are the potentials of the diagonal part of the $\mathrm{SU}(\mathrm{k})$ currents

$$
J_{\mu}^{i}=-\frac{1}{\sqrt{\pi}} \epsilon_{\mu}^{\nu} \partial^{\nu} \phi^{i},
$$

while $J_{\mu}=-\sqrt{\frac{k}{\pi}} \epsilon_{\mu \nu} \partial^{\nu} E$ is the $\mathrm{U}(1)$ current. 
One of the characteristics of of the Schwinger model with flavour is the fact that periodicity with respect to $\theta$ changes. Indeed, instead of being periodic under $\theta \rightarrow \theta+2 \pi$, the theory is rather symmetric under $\theta \rightarrow \theta+2 \pi / k$, where $k$ is the number of flavours. One can construct the following flavour-carrying fermionic operator [3]

$$
\mathcal{F}_{f}(z)=e^{i \sqrt{\pi} \gamma_{5} \chi_{f}+i \sqrt{\pi} \int_{x^{\prime}}^{\infty} d z^{1} \dot{\chi}_{f}\left(x^{0}, z^{1}\right)},
$$

in terms of which mesons $\mathcal{M}$ and baryons $\mathcal{B}$ are

$$
\mathcal{M}=\mathcal{F}_{f} \mathcal{F}_{f}^{\dagger}, \quad \mathcal{B}=\Pi_{i=0}^{k} \mathcal{F}_{f_{i}} .
$$

In the massless case, $\mathcal{F}_{f}$ are observable quantities which correspond to screened quarks. We verify that they carry $\mathrm{SU}(\mathrm{k})$ charge,

$$
\left[Q^{i}, \mathcal{F}_{f}^{ \pm}(x)\right]=-\tau_{f f}^{i} \mathcal{F}_{f}^{ \pm}(x)
$$

but no $\mathrm{U}(1)$ charge.

In the massive Schwinger model the situation changes again, because in general $\mathcal{F}_{f}^{ \pm}(x)$ does not commute with the mass term. Indeed

$$
\begin{aligned}
{\left[\mathcal{F}_{f}^{ \pm}(x),\right.} & \left.\sum_{f} \cos \left(2 \sqrt{\frac{\pi}{k}} E(y)+2 \sqrt{\pi} \chi_{f}(y)+\theta\right)\right] \\
= & \sum_{f}\left[\cos \left(2 \sqrt{\frac{\pi}{k}} E+2 \sqrt{\pi} \chi_{f}+\theta-\frac{2 \pi}{k} \Theta\left(y^{1}-x^{1}\right)\right)\right. \\
& \left.-\cos \left(2 \sqrt{\frac{\pi}{k}} E+2 \sqrt{\pi} \chi_{f}+\theta\right)\right] \mathcal{F}_{f}(x)
\end{aligned}
$$

This shows again that $\mathcal{F}_{f}$ does not generate an eigenstate of the Hamiltonian for non-vanishing fermion mass, unless $\theta=\pi / k$ where one can show that after a dressing of the operator $\mathcal{F}_{f}$ by the kink operator, it commutes with the mass term [4], and we are once more back to the screening situation.

\section{$3 \quad$ Non-abelian gauge theories}

Two-dimensional QCD with fermions in an arbitrary representation may be described in terms of quark fields $\psi_{i}^{f}$ where $i$ is the usual 
colour index and $f=1, \cdots, k$ is a flavour quantum number. The full QCD lagrangian is

$$
S=\int d^{2} x\left[-\frac{1}{4} \operatorname{tr} F_{\mu \nu} F^{\mu \nu}+\bar{\psi}_{i}^{f}\left(i \not \partial \delta^{i j}+e \not^{i j}\right) \psi_{j}^{f}-m^{\prime} \bar{\psi}^{f} \psi^{f}\right] .
$$

Although this model is two-dimensional, it is by no means simple and has, so far, evaded an exact solution. For an $\mathrm{SU}(\mathrm{N})$ gauge group and $k=1$ the large $\mathrm{N}$ limit was studied [11] and the approximate spectrum was obtained. Bosonisation of the fermionic determinant in terms of the Wess-Zumino-Witten functional helps understanding part of the structure of the theory [12]. That is, one can use the following identity:

$$
\int \mathcal{D} \bar{\psi} \mathcal{D} \psi e^{i \int d^{2} x \bar{\psi}(i \not D-m) \psi}=\int \mathcal{D} \hat{g} e^{i \Gamma[\hat{g}, A]+i m^{\prime 2} \int d^{2} x \operatorname{tr}\left(\hat{g}+\hat{g}^{-1}\right)},
$$

where the gauged Wess-Zumino-Witten action is

$$
\begin{aligned}
\Gamma[\hat{g}, A]= & \Gamma[\hat{g}]+\frac{1}{4 \pi} \int d^{2} x \operatorname{tr}\left[e^{2} A^{+} A_{+}-e^{2} A_{+} \hat{g} A_{-} \hat{g}^{-1}\right. \\
& \left.-i e A_{+} \hat{g} \partial_{-} \hat{g}^{-1}-i e A_{-} \hat{g}^{-1} \partial_{+} \hat{g}\right]
\end{aligned}
$$

and

$$
\begin{aligned}
\Gamma[g] & =\frac{1}{8 \pi} \int d^{2} x \operatorname{tr} \partial_{\mu} g^{-1} \partial^{\mu} g \\
& +\frac{1}{4 \pi} \operatorname{tr} \int_{0}^{1} d r \int d^{2} x \epsilon^{\mu \nu} \tilde{g}^{-1} \frac{d \tilde{g}}{d r} \tilde{g}^{-1} \partial_{\mu} \tilde{g} \tilde{g}^{-1} \partial_{\nu} \tilde{g}
\end{aligned}
$$

is the usual WZW action, and $\tilde{g}(r, x)$ interpolates a trivial configuration $\tilde{g}(0, x)=1$ to $g(x)$ itself, i.e. $\tilde{g}(1, x)=g(x)$. The mass parameter $m$ in the bosonic theory has been chosen with an arbitrary renormalisation and is related (but not necessarily equal) to the fermion mass $m^{\prime}$. The flavour $f$ in such a scheme is introduced in the usual manner. We decompose the gauge fields into the $\mathrm{SU}(\mathrm{N})$-valued fields $U$ and $V$, i.e.,

$$
A_{+}=\frac{i}{e} U^{-1} \partial_{+} U, \quad A_{-}=\frac{i}{e} V \partial_{-} V^{-1},
$$

such that

$$
\Gamma[\hat{g}, A]=\Gamma[U \hat{g} V]-\Gamma[U V]=\Gamma[g]-\Gamma[\tilde{\Sigma}],
$$

where $g=U \hat{g} V, \tilde{\Sigma}=U V$. The jacobian associated with transformations $(24)$ is non-trivial and contributes to the action with a term 
$-c_{v} \Gamma[\tilde{\Sigma}=U V]$. In the massless theory, the dressed field $g_{f}=U \hat{g}_{f} V$ decouples. This theory also contains the negative metric field $\Sigma$ (which is obtained from $\tilde{\Sigma}$ after some algebraic manipulations, $\Sigma=\beta \tilde{\Sigma}$ ) and the ghosts. The $g$ and $\Sigma$-fields together with the ghost system build up the vacuum structure of two-dimensional QCD. The selfinteraction term of the gauge field strength can be simplified: first we add a term $-\frac{1}{2}\left(E+\frac{1}{2} F_{+-}\right)^{2}$ to the lagrangian and then we define $\partial_{+}\left(U E U^{-1}\right)=\frac{i}{4 \pi} \beta^{-1} \partial_{+} \beta$. (These manipulations are described in detail in [7, 12].) Finally, we arrive at the effective action

$$
\begin{aligned}
S_{\mathrm{eff}}= & \sum_{f} \Gamma\left[g_{f}\right]-\left(c_{v}+k\right) \Gamma[\Sigma]+S_{\text {ghosts }}+k \Gamma[\beta] \\
& +\int d^{2} z \operatorname{tr}\left[\frac{1}{2}\left(\partial_{+} C_{-}\right)^{2}+i \lambda C_{-} \beta^{-1} \partial_{+} \beta\right] \\
& +m^{2} \int d^{2} z \operatorname{tr}\left[\sum_{f}\left(g_{f} \Sigma^{-1} \beta+g_{f}^{-1} \Sigma \beta^{-1}\right)\right],
\end{aligned}
$$

with $\lambda=\frac{e}{2 \pi}\left(c_{v}+k\right)$. The equations of motion read

$$
\begin{aligned}
\frac{1}{4 \pi} \partial_{+}\left(g_{f} \partial_{-} g_{f}^{-1}\right) & =m^{2}\left(g_{f} \Sigma^{-1} \beta-\beta^{-1} \Sigma g_{f}^{-1}\right), \quad f=1 \cdots k \\
-\frac{\left(c_{v}+k\right)}{4 \pi} \partial_{+}\left(\Sigma \partial_{-} \Sigma^{-1}\right) & =m^{2} \sum_{f=1}^{k}\left(\Sigma g_{f}^{-1} \beta^{-1}-\beta g_{f} \Sigma^{-1}\right) \\
-\frac{k}{4 \pi} \partial_{-}\left(\beta^{-1} \partial_{+} \beta\right) & +i \lambda\left[\beta^{-1} \partial_{+} \beta, C\right]+i \lambda \partial_{+} C \\
& =m^{2} \sum_{f=1}^{k}\left(g_{f} \Sigma^{-1} \beta-\beta^{-1} \Sigma g_{f}\right) \\
\partial_{+}^{2} C & =\lambda\left(\beta^{-1} i \partial_{+} \beta\right) .
\end{aligned}
$$

We proceed by considering the case of massless fermions in the fundamental representation $(k=1)$ and compute the inter-quark potential. We then introduce a pair of classical colour charges of strength $q^{a}$ and a distance $L$ apart. Such a pair is introduced in the action (26) by means of the substitution

$$
i\left(\beta^{-1} \partial_{+} \beta\right)^{a} \longrightarrow i\left(\beta^{-1} \partial_{+} \beta\right)^{a}-\frac{2 \pi}{e} q^{a}\left(\delta\left(x-\frac{L}{2}\right)-\delta\left(x+\frac{L}{2}\right)\right),
$$


where $a$ is a definite colour index. This adds the following new term to the action 2

$$
V(L)=\Delta S=S_{q}-S=-\left(c_{v}+1\right) q^{a}\left(C_{-}^{a}(L / 2)-C_{-}^{a}(-L / 2)\right) .
$$

The equation of motion for $C^{a}$ is now replaced by

$$
\partial_{+}^{2} C^{a}=i \lambda\left(\beta^{-1} \partial_{+} \beta\right)^{a}-\left(c_{v}+1\right) q^{a}\left(\delta\left(x-\frac{L}{2}\right)-\delta\left(x+\frac{L}{2}\right)\right),
$$

which implies, upon substitution into the $\beta$-field equation of motion,

$$
\begin{aligned}
\partial_{+}( & \left.\frac{i}{4 \pi \lambda} \partial_{-} \partial_{+} C+\left[\partial_{+} C, C\right]+i \lambda C\right)= \\
& \left(\frac{-i q^{a}}{2 e} \partial_{-}+\left(c_{v}+1\right)\left[q^{a}, C\right]\right)\left[\delta\left(x-\frac{L}{2}\right)-\delta\left(x+\frac{L}{2}\right)\right] .
\end{aligned}
$$

As we are fixing the external charge in the colour space, we propose the ansatz $C^{a}=q^{a} f(x)$, which renders the problem essentially abelian. As a result we find the following solution for the potential (32):

$$
V(L)=\frac{\left(c_{v}+1\right) \sqrt{\pi}}{2} \frac{q^{2}}{e}\left(1-e^{-2 \sqrt{\pi} \lambda L}\right)
$$

which implies a screening phase.

A more general discussion is, however required. Several points have been discarded in the previous discussion. First, we did not take account of the vacuum structure. In fact, two dimensional gauge theories may have a non-trivial $\theta$-vacuum which arises from charges placed at infinity [13], or from further degeneracies related [14] to the BRST structure of the massless fields and ghost system [15]. In the massless case these issues are rather marginal. However, they may play an important rôle in the massive case where the fields, which previously described only the vacuum structure, now effectively couple to the $\beta$-sector. This enlarges the physical subspace [16].

Let us, again, consider the one flavour theory (i.e. the fundamental representation for the fermions). Since, in effect, we will be solving the equivalent mechanical problem, we may rewrite the theory in terms of scalars rather than matrix-valued fields, as follows:

$$
g=e^{i 2 \sqrt{\pi} \varphi \sigma_{2}}, \quad \beta=e^{i 2 \sqrt{\pi} E \sigma_{2}}, \quad \Sigma=e^{-i 2 \sqrt{\pi} \eta \sigma_{2}},
$$

\footnotetext{
${ }^{2}$ This corresponds to minus the same term added to the hamiltonian.
} 
where the external charges are taken in the direction $\sigma_{2}$ in the $\mathrm{SU}(2)$ spaces. This parametrisation can be simply extended to the case of a general gauge group $\mathrm{SU}(\mathrm{N})$. In the massive theory, the equations of motion are

$$
\begin{aligned}
\partial_{+} \partial_{-} \varphi= & -4 \sqrt{\pi} m^{2} \sin 2 \sqrt{\pi}(E+\varphi+\eta) \\
\partial_{+} \partial_{-} \eta= & \frac{4 \sqrt{\pi}}{c_{v}+1} m^{2} \sin 2 \sqrt{\pi}(E+\varphi+\eta) \\
\partial_{+} \partial_{-} E+4 \pi \lambda^{2} E= & -4 \sqrt{\pi} m^{2} \sin 2 \sqrt{\pi}(E+\varphi+\eta) \\
& -2 \sqrt{\pi}\left(c_{v}+1\right) \lambda q\left[\Theta\left(x+\frac{L}{2}\right)-\Theta\left(x-\frac{L}{2}\right)\right]
\end{aligned}
$$

We notice the existence of the massless combination

$$
\partial_{+} \partial_{-}\left(\varphi+\left(c_{v}+1\right) \eta\right)=0
$$

which is, in fact, a remnant of the vacuum structure in the massive case. Moreover, in order to compute the potential we use the static limit where one discards the time derivatives in the above field equations. This is equivalent to considering the effective lagrangian

$$
\begin{aligned}
\mathcal{L}= & \frac{1}{2} \varphi^{\prime 2}-\frac{1}{2}\left(c_{v}+1\right) \eta^{\prime 2}+\frac{1}{2} E^{\prime 2}-2 m^{2} \cos 2 \sqrt{\pi}(\varphi+\eta+E) \\
& +2 \pi \lambda^{2} E^{2}+2 \sqrt{\pi}\left(c_{v}+1\right) \lambda E q\left[\Theta\left(x+\frac{L}{2}\right)-\Theta\left(x-\frac{L}{2}\right)\right] \\
& -\frac{\left(c_{v}+1\right)^{2} q^{2}}{2}\left[\Theta\left(x+\frac{L}{2}\right)-\Theta\left(\frac{x-L}{2}\right)\right] \\
= & \frac{1}{2} E^{\prime 2}+\frac{1}{2} \frac{c_{v}+1}{c_{v}} \Phi^{\prime 2}+2 \pi \lambda^{2} E^{2}-2 m^{2} \cos 2 \sqrt{\pi}(\Phi+E) \\
& +2 \sqrt{\pi}\left(c_{v}+1\right) \lambda q E\left[\Theta\left(x+\frac{L}{2}\right)-\Theta\left(x-\frac{L}{2}\right)\right] \\
& -\frac{\left(c_{v}+1\right)^{2} q^{2}}{2}\left[\Theta\left(x+\frac{L}{2}\right)-\Theta\left(x-\frac{L}{2}\right)\right]-\frac{\psi^{\prime 2}}{2 c_{v}},
\end{aligned}
$$

where $\Phi=\varphi+\eta$ and $\psi=\varphi+\left(c_{v}+1\right) \eta$. This change of variables is possible because only some of the contributions will couple to the

\footnotetext{
${ }^{3} \sigma_{i}$ are the Pauli matrices.

${ }^{4}$ We leave the Casimir $c_{v}$ as a free parameter, since the expressions corresponding to the Schwinger model will simply be obtained from the $\mathrm{SU}(\mathrm{N})$ model by taking the limit $c_{v} \rightarrow 0$.
} 
physical variables. In addition, we find instanton-like solutions, where the field $E$ asymptotically vanishes as $x \rightarrow \pm \infty$ and makes a lump within the range $|x|<L / 2$. Thus the cosine term may be expanded. Later, we have to confirm that the solution is consistent with such a condition.

It is worth remaking that the abelian case $\left(c_{v} \rightarrow 0\right)$ corresponds to a rather singular limit in the lagrangian (41) where the kinetic term for $\Phi$ as well as for $\psi$ have each a divergent coefficient. We will see later that at the level of interacting potential this limit is smooth.

In the weak-limit approximation, we expand the cosine term. Consequently, we diagonalise the hamiltonian and solve the equations of motion ${ }^{5}$.

The diagonalisation of the quadratic lagrangian leads to the expression

$$
\begin{aligned}
\mathcal{L} & =-\frac{1}{2 c_{v}} \psi^{\prime 2} \\
& +\left(1+\epsilon a^{2}\right)\left\{\frac{1}{2} \chi_{+}^{\prime 2}+\frac{1}{2} m_{+}^{2} \chi_{+}^{2}+\lambda Q_{+} \chi_{+}\right\} \\
& +\frac{\left(1+\epsilon a^{2}\right)}{a^{2}}\left\{\frac{1}{2} \chi_{-}^{\prime 2}+\frac{1}{2} m_{-}^{2} \chi_{-}^{2}+\lambda Q_{-} \chi_{-}\right\}
\end{aligned}
$$

where we have found it useful to define the following variables :

$$
\begin{aligned}
& \chi_{+}=\frac{1}{1+\epsilon a^{2}}(E-a \Phi), \\
& \chi_{-}=\frac{1}{1+\epsilon a^{2}}(\Phi+\epsilon a E)
\end{aligned}
$$

and the parameters :

$$
\begin{aligned}
\epsilon & =\frac{c_{v}}{\left(c_{v}+1\right)}, \\
a & =-\frac{8 \pi m^{2}}{m_{+}^{2}-16 \epsilon m^{2}} \\
m_{ \pm}^{2} & =2 \pi\left[\left(\lambda^{2}+(1+\epsilon) 2 m^{2}\right) \pm \sqrt{\left(\lambda^{2}+(1+\epsilon) 2 m^{2}\right)^{2}-8 \epsilon \lambda^{2} m^{2}}\right]
\end{aligned}
$$

\footnotetext{
${ }^{5}$ All the forthcoming computations will in general be valid for any compact group. In such cases, the mass term can always be expanded in terms of algebra-valued fields after a convenient parametrisation.
} 


$$
\begin{aligned}
Q_{ \pm} & =q_{ \pm}\left[\Theta\left(x-\frac{L}{2}\right)-\Theta\left(x+\frac{L}{2}\right)\right] \\
q_{+} & =\frac{2 \sqrt{\pi}\left(c_{v}+1\right) q}{\left(1+\epsilon a^{2}\right)} \\
q_{-} & =\frac{2 \sqrt{\pi} \epsilon a\left(c_{v}+1\right) q}{\left(1+\epsilon a^{2}\right)} .
\end{aligned}
$$

Solving the corresponding equations of motion yields:

$$
\chi_{ \pm}=\frac{\lambda q_{ \pm}}{m_{ \pm}^{2}} \begin{cases}\sinh \left(m_{ \pm} \frac{L}{2}\right) e^{-m_{ \pm}}|x| & |x|>\frac{L}{2} \\ \left(1-e^{-m_{ \pm} L / 2} \cosh m_{ \pm} x\right) & |x|<\frac{L}{2}\end{cases}
$$

from which we obtain the inter-quark potential energy

$$
\begin{aligned}
& V(L)=\frac{\left(c_{v}+1\right)^{2} q^{2}}{2} \\
& \quad \times\left[\left(\frac{4 \pi \lambda^{2}-m_{-}^{2}}{m_{+}^{2}-m_{-}^{2}}\right)\left(\frac{1-e^{-m_{+} L}}{m_{+}}\right)+\left(\frac{m_{+}^{2}-4 \pi \lambda^{2}}{m_{+}^{2}-m_{-}^{2}}\right)\left(\frac{1-e^{-m_{-} L}}{m_{-}}\right)\right] .
\end{aligned}
$$

Thus we find two mass scales given by $m_{+}$and $m_{-}$. Both of these lead to screening-type contributions.

Next, we compare the results with those obtained for the Schwinger model. In the abelian case, the combination of the matter boson $\varphi$ and the negative metric scalar $\eta$ gives rise to the $\theta$-angle. That is, the combination

$$
\Phi \equiv \varphi+\eta=\theta
$$

appears in the mass term. When fermions are massless, the electric field and the matter boson decouple. However, due to a Higgs mechanism, the electric field acquires a mass and, therefore, a long-range force does not exist. This leads to a pure screening potential. On the other hand, for massive fermions, the electric field couples to the matter boson. Yet, $\Phi$ remains massless. The coupling through the mass is the origin of the long-range force in the massive $\mathrm{U}(1)$ case. The potential is confining.

On the contrary, the expression (52) for the potential indicates the absence of a long-range force in the non-abelian theory. This can be understood by recalling that there are two independent and relevant combinations of the fields $\varphi$ and $\eta$. The massless combination, which 
describes the vacuum constraints, is

$$
\psi \equiv \varphi+\left(c_{v}+1\right) \eta
$$

This combination decouples from the electric field (see eqn. (39)). The other one, $\Phi \equiv \varphi+\eta$, which is massive, is the combination that couples to $E$. Therefore, as both $E$ and $\Phi$ are massive, there is no long-range force. This is confirmed by our explicit computations.

The abelian potential (7) can also be obtained from (52) by taking the limit $c_{v} \rightarrow 0$. In this limit, the mass scale $m_{-}$tends to zero and we recover the confinement term.

It is interesting to examine the behaviour of the screening potential (52) in extreme limits. In the strong coupling regime, $\lambda^{2}>>m^{2}$, the mass parameter $m_{+}$dominates $\left(m_{+}>>m_{-}\right)$and we have

$V(L)_{(m<<e)} \simeq \frac{\left(c_{v}+1\right)^{2} q^{2}}{2}\left\{\frac{\left(1-e^{-2 \sqrt{\pi} \lambda L}\right)}{2 \sqrt{\pi} \lambda}+\frac{\sqrt{\pi \epsilon} m}{\lambda^{2}}\left(1-e^{-2 \sqrt{2 \pi \epsilon} m L}\right)\right\}$.

On the other hand, in the weak coupling limit, $m>>e$, we obtain

$$
V(L)_{(m>>e)} \simeq \frac{\left(c_{v}+1\right) q^{2}}{4 \sqrt{\pi} \lambda} \sqrt{\frac{1+\epsilon}{\epsilon}}\left(1-e^{-2 \sqrt{\pi \epsilon /(1+\epsilon)} \lambda L}\right) .
$$

In both regimes, the potential is governed by the parameter $\lambda$, i.e. by the coupling constant.

\section{Higher representations}

So far, we have considered fermions in the fundamental representation. In this section, we continue our quest for confinement by reformulating the preceding analysis using fermions in higher representation [8]. This can be done by introducing copies of the fermionic fields, that is

$$
\mathcal{L}_{\mathrm{fermi}}=\sum_{f}\left(\bar{\psi}_{f} i \not D \psi_{f}-m^{\prime} \bar{\psi}_{f} \psi_{f}\right)
$$

This implies that the bosonised version of theory contains a set of fields $g_{f}$, each of them in the fundamental representation.

Following the procedure of the last section, we fix the external charges in colour space, parametrise the fields as in equation (36) and 
take the weak-field and static limit. Finally, we arrive at the following diagonalised lagrangian 우

$$
\begin{aligned}
\mathcal{L} & =\sum_{f=1}^{k}\left[\frac{1}{2}\left(\partial_{\mu} \zeta_{f}\right)^{2}-4 \pi m^{2} \zeta_{f}^{2}\right]-\frac{1}{2 c_{v}} \psi^{\prime 2} \\
& +k\left(1+\epsilon a^{2}\right)\left\{\frac{1}{2} \chi_{+}^{\prime 2}+\frac{1}{2} m_{+}^{2} \chi_{+}^{2}+\lambda Q_{+} \chi_{+}\right\} \\
& +\frac{k\left(1+\epsilon a^{2}\right)}{a^{2}}\left\{\frac{1}{2} \chi_{-}^{\prime 2}+\frac{1}{2} m_{-}^{2} \chi_{-}^{2}+\lambda Q_{-} \chi_{-}\right\},
\end{aligned}
$$

where the decoupled fields are

$$
\begin{array}{rlrl}
\zeta_{f} & =\left(\varphi_{f}+\eta\right)-\Phi, & \sum_{f=1}^{k} \zeta_{f}=0, \\
\Phi & =\frac{1}{f} \sum_{f=1}^{k}\left(\varphi_{k}+\eta\right), \\
\psi & =\sum_{f=1}^{k} \varphi_{f}+\left(c_{v}+k\right) \eta
\end{array}
$$

and $\chi_{ \pm}$were defined in (43) and (44). The parameters are

$$
\begin{aligned}
\epsilon & =\frac{c_{v}}{\left(c_{v}+k\right)} \\
m_{ \pm}^{2} & =\frac{2 \pi}{k}\left[\left(\lambda^{2}+(1+\epsilon) 2 k m^{2}\right) \pm \sqrt{\left(\lambda^{2}+(1+\epsilon) 2 k m^{2}\right)^{2}-8 \epsilon k \lambda^{2} m^{2}}\right] \\
q_{+} & =\frac{2 \sqrt{\pi}\left(c_{v}+k\right) q}{k\left(1+\epsilon a^{2}\right)} \\
q_{-} & =\frac{2 \sqrt{\pi} \epsilon a\left(c_{v}+k\right) q}{k\left(1+\epsilon a^{2}\right)}
\end{aligned}
$$

with $a$ and $Q_{ \pm}$given by (46) and (48). The finite-energy solutions to the equations of motion should tend to the corresponding vacuum states at $x= \pm \infty$. These vacuum states are obtained by extremising the full expression for the potential (prior to taking the weak-field

\footnotetext{
${ }^{6}$ Note that now $g_{f}=e^{i \varphi_{f} \sigma_{2}}, f=1, \cdots, k$.
} 
limit). This leads to the condition $E( \pm \infty)=07$ and two possibilities for the field $\Phi ; \Phi( \pm \infty)=0$ or $\Phi( \pm \infty)=\sqrt{\pi}$. However, only the first choice yields a finite-energy solution, which in turn implies $\chi_{ \pm}( \pm \infty)=0$. The finite-energy solutions obey the boundary condition $\chi_{ \pm}( \pm \infty)=0$ and the same expressions as (51), but with the above parameters. The inter-quark potential is

$$
\begin{aligned}
& V(L)=\frac{\left(c_{v}+k\right)^{2} q^{2}}{2 k} \\
& \quad \times\left[\left(\frac{4 \pi \lambda^{2}-k m_{-}^{2}}{m_{+}^{2}-m_{-}^{2}}\right)\left(\frac{1-e^{-m_{+} L}}{m_{+}}\right)+\left(\frac{k m_{+}^{2}-4 \pi \lambda^{2}}{m_{+}^{2}-m_{-}^{2}}\right)\left(\frac{1-e^{-m_{-} L}}{m_{-}}\right)\right]
\end{aligned}
$$

We have verified that further solutions obtained by means of different boundary conditions do not lead to confinement. To explain this result, we take up the example of $k=2$ ?. We define $\phi_{f}=\varphi_{f}+\eta$. The saddle points of the potential correspond to $E=0$ and either $\phi_{f}=0$ or $\phi_{f}=\sqrt{\pi}$. We consider one of the $\phi_{f}$ s to be in the second vacuum state. For instance, we take $\phi_{1}( \pm \infty)=0$ and $\phi_{2}( \pm \infty)=\sqrt{\pi}$. The non-trivial choice for $\phi_{2}$ corresponds to a non-trivial $\theta$ vacuum and is equivalent to a change of sign in its mass term $\left(m^{2} \rightarrow-m^{2}\right)$. In the weak-field limit we obtain the following equations of motion:

$$
\begin{aligned}
-E^{\prime \prime}+4 \pi \lambda^{2} E+8 \pi m^{2}\left(\phi_{1}-\phi_{2}\right) & =-2 \sqrt{\pi}\left(c_{v}+2\right) \lambda q \\
\times\left[\Theta\left(x+\frac{L}{2}\right)\right. & \left.-\Theta\left(x-\frac{L}{2}\right)\right], \\
-\frac{c_{v}+1}{c_{v}} \phi_{1}^{\prime \prime}-\frac{1}{c_{v}} \phi_{2}^{\prime \prime}+8 \pi m^{2}\left(\phi_{1}+E\right) & =0, \\
-\frac{c_{v}+1}{c_{v}} \phi_{2}^{\prime \prime}-\frac{1}{c_{v}} \phi_{1}^{\prime \prime}-8 \pi m^{2}\left(\phi_{2}+E\right) & =0 .
\end{aligned}
$$

Thus, we have a system of coupled differential equations. These lead to three different values of the mass-squared parameters which correspond to three "Higgs" masses. One of these is negative and, therefore, leads to an oscillatory term. We find no solutions which obey all the constraints (i.e. which are asymptotically finite, continuous and have continuous derivatives at the positions of the quarks). Therefore, screening is the only phase of the theory.

\footnotetext{
${ }^{7}$ Recall that in the abelian case we had many options for the field E depending on the value of $\theta$, as in eqn. (8).

${ }^{8}$ The generalisation to a generic $k$ is trivial.
} 
We conclude this section by justifying some of the approximations we have used so far. Both in the Schwinger and the non-abelian models we have assumed that in expanding the cosine term $(\cos 2 \sqrt{\pi}(E+$ $\Phi)$ ), which appears in the action (3), one can discard higher order contributions. The solutions, subsequently, found have the following property:

$$
|2 \sqrt{\pi}(E+\Phi)(x)| \leq \frac{2 \pi q}{e}\left|\frac{(1+a)(1-\epsilon a)}{\left(1+\epsilon a^{2}\right)}\right|\left(e^{-m_{-} L / 2}-e^{-m_{+} L / 2}\right) .
$$

In the non-abelian case, where both mass scales $m_{ \pm}$are non-vanishing the right-hand-side of the above equation tends to zero as $L$ tends to infinity. Therefore, the larger the inter-quark distance, the better our approximation.

The abelian limit is rather different: when $c_{v}$ approaches 0 , the small mass $m_{-}$tends to zero and this yields

$$
|2 \sqrt{\pi} E(x)| \leq \frac{2 \pi q}{e} \frac{\lambda^{2}}{\lambda^{2}+2 m^{2}}\left(1-e^{-\alpha L / 2}\right) .
$$

Hence, in the Schwinger model, the approximations hold only if the fermion mass is much larger than the coupling constant. Moreover, for large separations and nonvanishing fermion mass, we have to take into account the confinement term, which blows up for large distances.

\section{$5 \quad$ Exotic states}

In the preceding sections, we have presented a semi-classical analysis of two-dimensional QCD. In order to distinguish between the screening and the confinement phases, we have used a dipole-dissociation test. If the particles are confined, an infinite amount of energy is required to isolate them. In this case, as the inter-quark distance increases pair production occurs which obscures the physical interpretation of the results. On the other hand, in the screening phase the amount of energy required to dissociate the dipole is finite. Although charge (or colour) cannot be seen because of vacuum polarisation, further structures ( or quantum numbers) can be observed.

In this section, we outline the construction of eigenstates of the hamiltonian which carry flavour quantum numbers. These are the 
analogues of the exotic states in the Schwinger model (see Section 2). This provides a more elaborate confirmation of the screening phase.

We construct the exotic states by means of the fermionic operator 此.

$$
\mathcal{F}_{f}(x)=\prod_{a} e^{i \sqrt{\pi} \phi_{f}^{a}\left(x^{0}, x^{1}\right) /\left(c_{v}+k\right)+i\left(c_{v}+k\right) \sqrt{\pi} \int_{-\infty}^{x^{1}} \dot{\phi}_{f}^{a}\left(x^{0}, y^{1}\right) d y^{1}}=\prod_{a} \mathcal{F}_{f}^{a},
$$

where the field $\phi_{f}^{a}$ does not carry colour charge. 9 From the semiclassical discussion, the combination $\left(\varphi_{f}^{a}+\eta\right)$ is the natural candidate for the operator $\phi_{f}^{a}$. This is because we have chosen symmetric boundary conditions $\left(\phi_{f}^{a}(+\infty)=\phi^{a}(-\infty)\right)$ which imply that $\phi_{f}^{a}$ carries no charge. In the quantum theory [16], the operator $\phi_{f}^{a}=\varphi_{f}^{a}+\eta$ appears in the BRST current

$$
J_{+}=c_{+}\left(i g_{f}^{-1} \partial_{+} g_{f}-i\left(c_{v}+k\right) \Sigma^{-1} \partial_{+} \Sigma+\text { ghosts }\right),
$$

which is conserved (actually vanishing) and leads to the topological charges

$Q=\left(\sum_{f=1}^{k} \varphi_{f}+\left(c_{v}+k\right) \eta\right)(t, \infty)-\left(\sum_{f=1}^{k} \varphi_{f}+\left(c_{v}+k\right) \eta\right)(t,-\infty)+\cdots$

where $\cdots$ stands for the commutator-type corrections.

Next, we argue that the operator (72) commutes with the mass term. We use the parametrisation

$$
\begin{aligned}
g & =e^{i \varphi^{1} \sigma^{1}} e^{i \varphi^{2} \sigma^{2}} e^{i \varphi^{3} \sigma^{3}}, \\
\Sigma & =e^{-i \eta^{1} \sigma^{1}} e^{-i \eta^{2} \sigma^{2}} e^{-i \eta^{3} \sigma^{3}},
\end{aligned}
$$

in the $\mathrm{SU}(\mathrm{N})$ model and take the commutator of $\mathcal{F}_{f}$ with the mass term. This shifts $\varphi^{a}$ by $2 \pi\left(c_{v}+k\right)$, and $\eta^{a}$ by $2 \pi$. Since $\mathrm{SU}(2)$ is a compact group, we conclude that $\mathcal{F}_{f}$ commutes with the hamiltonian. This result can be generalised to any $\mathrm{SU}(\mathrm{N})$ gauge group.

By comparing expression (72) with the fermionic operator (16), we see that the field $\eta$ plays a rôle similar to that played previously by

\footnotetext{
${ }^{9}$ We do not expect (72) to be the complete operator which describes flavoured physical states. Corrections involving multiple commutators, due to the non-abelian character of the theory, can appear.
} 
the sum $\sum_{i=1}^{k} \psi_{k}$ in the abelian theory. Consequently, the fields are not constrained in the non-abelian model and enjoy canonical commutation relations. Thus, kink dressing might be needed [2], [3]. In addition, the $\theta$-vacuum does not enter the expression for the fermionic operator (72) in the non-abelian theory.

\section{Conclusion}

Having used the semi-classical methods, we conclude that two dimensional QCD displays screening even when the fermions are massive.

An important rôle in our calculation has been played by the combination of the bosonised matter and negative metric field (i.e. $\Phi=$ $\varphi+\eta)$. Since $\Phi$ is now a dynamical field, the effect of a possible $\theta$-parameter or of a background BRST charge is immaterial. Furthermore, $\Phi$ has no (colour) topological number, which is essential for the construction of colourless states with flavour degrees of freedom.

In the Schwinger model (as well as in QCD on the SU(N) torus 21]) such states are-in some particular $\theta$-worlds-the remnants of quark states. The $\mathrm{U}(1)$ charge is screened by the gauge field interaction. Nevertheless, a shadow of its presence is left on the multiplicative quantum number of the exotic states. For a very refined discussion of the problem of screening and confinement in QED see ref. 22].

In two-dimensional QCD, we are faced with a similar situation. A well-defined colourless fermionic operator can be constructed and thus screening is unavoidable. Presumably, the disappearance of colour can be understood in terms of the spurionisation idea which is equivalent to superconductive condensation [17, 20]. This phenomenon is expressed by the non-vanishing expectation value of the dressed fermionic field [10]. Therefore, we search for locally gauge-invariant fields which have non-zero expectation values. These gauge-invariant quantities can be obtained by dressing the fermionic fields with gauge field flux tubes. In our bosonised approach, such a dressing is done by using the gauge potentials $U$ and $V$.

Finally a few words of caution. we have treated the fermion mass perturbatively. For large fermion masses, the fields obey sine-Gordonlike equations which deserve further studies.

Also, it is known that in the Wilson-loop approach the perimeter 
versus area law criteria may fail to distinguish between screening and confinement, due to pair production. The decisive test for screening is the existence of the exotic states which we have considered. The "baryon" (see Section 5) is the best probe of the screening mechanism. Although our construction is rather robust and gives the correct results in all known limits, we believe it is worthwhile to explicitly construct the exotic states in higher representations. Unfortunately, only a few results are available and these are mainly for massless fields [19].

In addition, it is worth investigating into the discrepancy which exists between different results concerning the fermion condensates in QCD with adjoint fermions [18].

Finally, it would be interesting to consider the analogue of our analysis in 4 dimensional QCD. If our result persists in $3+1$ dimensions it would be crucial to understand Seiberg-Witten's monopole condensation in supersymmetric Yang-Mills theory, where such a condensation is triggered by a mass parameter [9].

\section{Acknowledgements}

We thank I. Klebanov for discussions, and K. D. Rothe for the critical reading of the manuscript.

\section{References}

[1] J. Schwinger, Phys. Rev. Lett. 3 (1959) 1296.

J. Lowenstein and J. A. Swieca, Annals of Phys. 68 (1971) 172.

A. Cashen, J. Kogut and L. Susskind, Phys. Rev. D10 (1974) 732 .

[2] H. J. Rothe, K. D. Rothe and J. A. Swieca, Phys. Rev. D19 (1979) 3020.

[3] K. D. Rothe, B. Schroer, Nucl. Phys. B185 (1981) 429, Nucl. Phys. B172 (1980) 383.

[4] E. Abdalla, M. C. Abdalla and K. D. Rothe, " Non-perturbative methods in two dimensional quantum field theory", World Scien- 
tific, 1991.

[5] S. Coleman, Annals of Physics 101 (1976) 239.

[6] J. Ellis, Y. Frishman, A. Hanany and M. Karliner, Nucl. Phys. B389 (1992) 189.

[7] E. Abdalla and M. C. B. Abdalla, Phys. Rep. 265 (1996) 253.

[8] D. J. Gross, I. R. Klebanov, A. V. Matytsin and A. V. Smilga, Preprint, "Screening vs Confinement in $1+1$ dimensions", hepth/9511104.

[9] N. Seiberg and E. Witten, Nucl. Phys. B426 (1994) 19.

[10] V. Kurak, B. Schroer and J. A. Swieca, Nucl. Phys. B134 (1978) 61.

[11] G. 't Hooft, Nucl. Phys. B75 (1974) 461.

C. G. Callan, N. Coote and D. J. Gross, Phys. Rev. D13 (1976) 1649 .

[12] E. Abdalla and M. C. B. Abdalla, Int. J. Mod. Phys. A10 (1995) 1611 .

[13] E. Witten, Nuovo Cimento A51 (1979) 325.

[14] E. Abdalla and K. D. Rothe Phys. Lett 363B (1995) 85

[15] D. Karabali and H. J. Schnitzer, Nucl. Phys. B329 (1990) 649.

[16] E. Abdalla, M. C. B. Abdalla and K. D. Rothe, Preprint, hepth/9511191.

[17] H. Nielsen and Schroer, Nucl. Phys. B120, (77) 61. 
[18] A. Smilga, Phys. Rev. D49 (1994) 683.

[19] S. G. Naculich, H. J. Schnitzer, Nucl. Phys. B347 (1990) 687.

[20] K. D. Rothe and J. A. Swieca, Phys. Rev. D15 (77) 541.

[21] L. V. Belvedere, K. D. Rothe, B. Schroer and J. A. Swieca Nucl. Phys. B153 (1979) 112.

[22] B. Schroer Rev. Math Phys. 7 (1995) 645, K. Fredenhagen in Erice School on Mathematical Physics (1985) 265. 